\title{
Acute Ischaemic Stroke Secondary to a Mobile Thrombus in the Common Carotid Artery - Case Report
}

\author{
Zoltán Bajkó ${ }^{1,2}$, Smaranda Maier ${ }^{1,2}$, Silvia Rusu2 ${ }^{2}$ Anca Moțățăianu ${ }^{1,2^{*}}$ \\ ${ }^{1}$ University of Medicine and Pharmacy Târgu Mureș, Department of Neurology, Gheorghe Marinescu street 38, \\ 540139 Târgu Mureș, Romania \\ 2 Mureș County Clinical Emergency Hospital Târgu Mureș, Neurology Clinic I, Gheorghe Marinescu street 50, 540136, \\ Târgu Mureș, Romania
}

\begin{abstract}
A mobile thrombus in the carotid arteries is a very rare ultrasonographic finding and is usually diagnosed after a neurological emergency, such as a transient ischemic attack or cerebral infarction. We present the case of a 54-year-old man with vascular risk factors (a heavy smoker, untreated hypertension) who was admitted to the emergency unit with right sided hemiparesis and aphasia. A cerebral CT scan showed a left middle cerebral artery territory infarction. The duplex ultrasound examination revealed mild atherosclerotic changes in the right common and internal carotid arteries, right-sided complete subclavian steal phenomenon and a complicated hypoechoic atherosclerotic plaque in the left common carotid artery with a large mobile thrombus. Due to the high embolization risk, the patient was hospitalised and prescribed Aspirin together with low molecular weight Heparin. We recorded an improvement in the patient's neurological status and the control duplex scan revealed disappearance of the thrombus. The presence of floating thrombus in a patient with clinical and imagistic evidence of stroke is a major therapheutic challenge for the neurologist. The treatment strategies are not standardized and must be individualized, however in our case parenteral anticoagulation proved to be successful.
\end{abstract}

Keywords: thrombus, carotid, emboli

Received: 02 January 2015 / Accepted: 15 January 2015

\section{INTRODUCTION}

A mobile thrombus in the carotid arteries is a very rare ultrasonographic finding and is usually diagnosed after a neurological emergency, such as a transient ischemic attack or cerebral infarction [1]. According to Bhatti et al., the ideal definition of a mobile thrombus is "an elongated thrombus attached to the arterial wall with circumferential blood flow at its distal most aspect with cyclical motion relating to cardiac cycles" [2].

\section{CASE REPORT}

A 54-year-old man with vascular risk factors (a heavy smoker, untreated hypertension) was admitted to the emergency unit with right sided hemiparesis and apha- sia. Admission was more than twelve hours after onset of the symptoms.

Neurological examination revealed a grade 1 rightsided hemiparesis and severe aphasia.

A cerebral CT scan showed a left middle cerebral artery territory infarction (Figure 1).

The duplex ultrasound examination revealed mild atherosclerotic changes in the right common and internal carotid arteries, right-sided complete subclavian steal phenomenon and a complicated hypoechoic atherosclerotic plaque in the left common carotid artery with a large mobile thrombus (Figure 2, 3).

The laboratory data showed all test values to be within in the normal range. Cardiac embolic source was ruled out.

\footnotetext{
* Correspondence to: Anca Moțățăianu, University of Medicine and Pharmacy Tîrgu Mureș, Gheorghe Marinescu street 50, 540136, Târgu Mureș, Romania Tel/fax 0265214510; e-mail: iipascu@redatronic.ro
} 


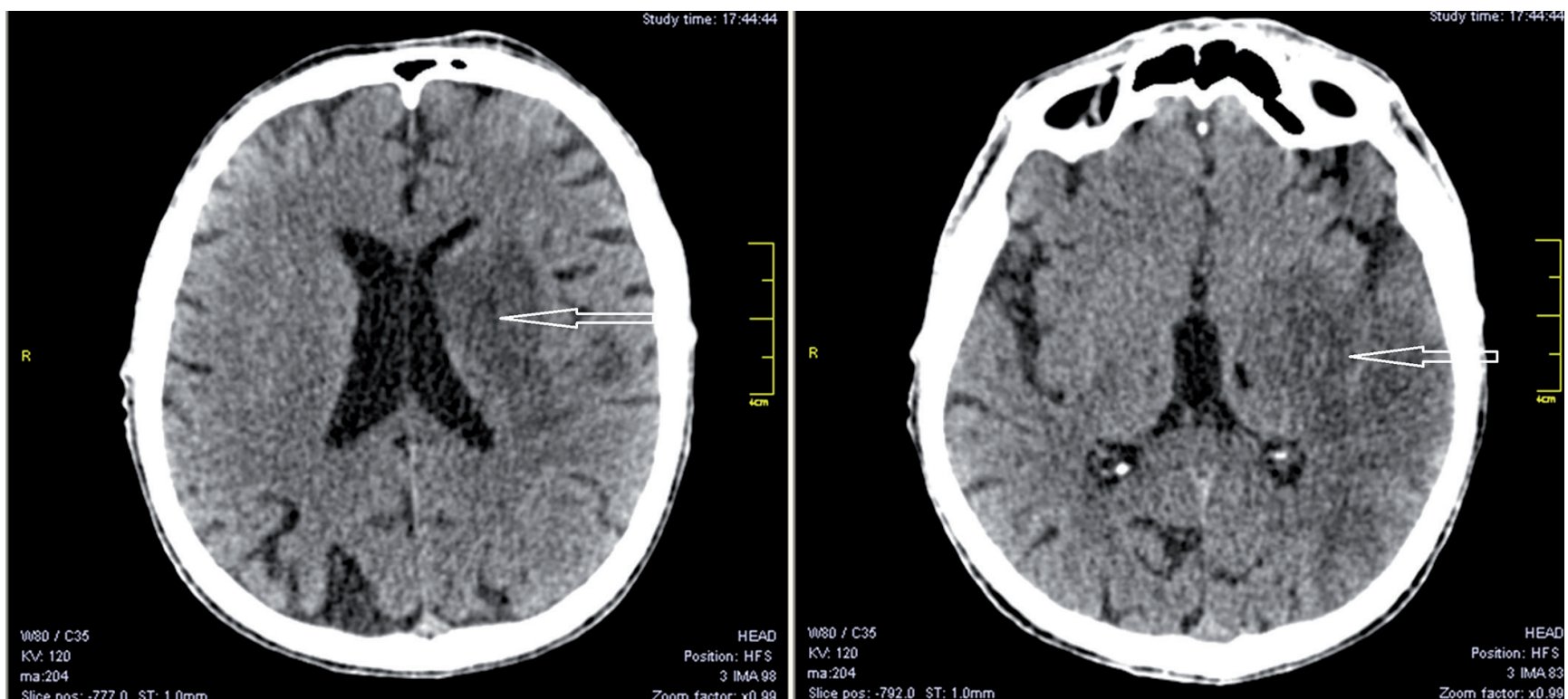

Figure 1. Axial non-contrast CT scan of the brain demonstrating a left middle cerebral artery infarction (arrow).
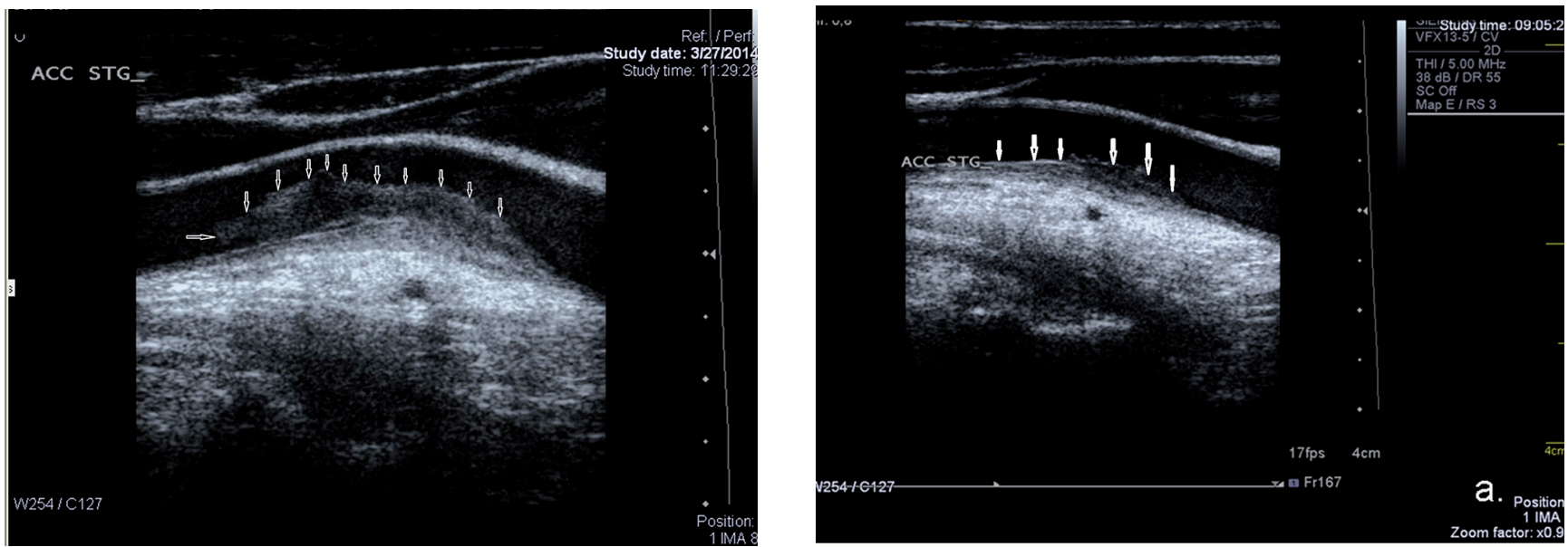

Figure 2. B-mode ultrasound examination, longitudinal scan of the left common carotid artery showing a mobile thrombus (arrows).

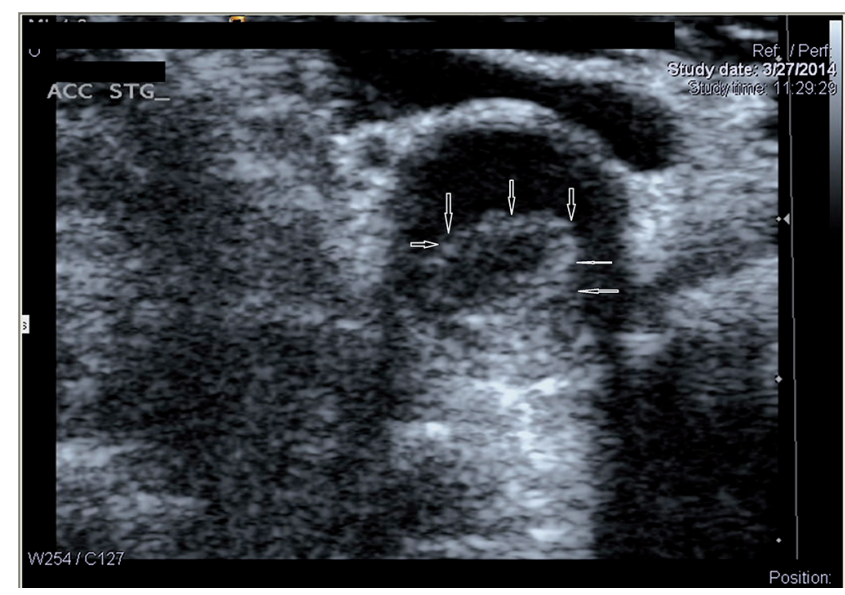

Figure 3. B-mode ultrasound examination, axial scan of the left common carotid artery showing a mobile thrombus (arrows).

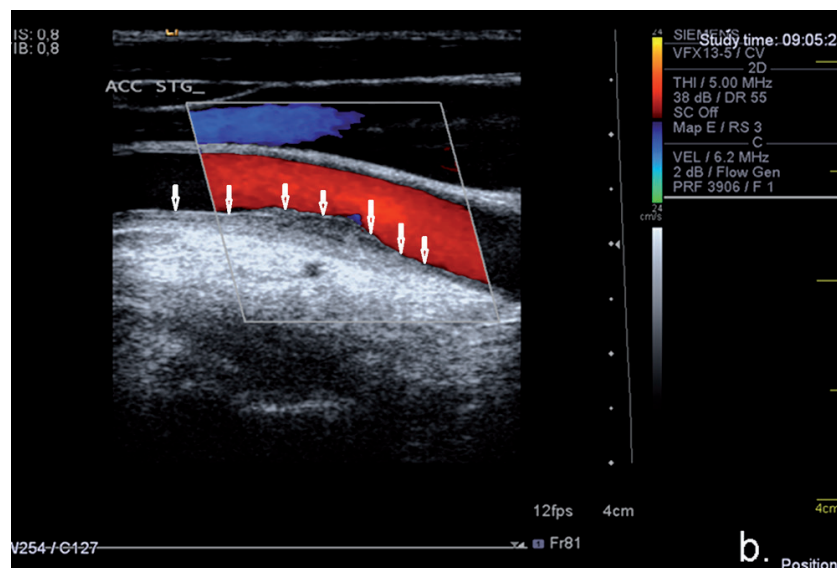

Figure 4. Control B-mode (a) and color mode (b) ultrasound examination, longitudinal scan of the left common carotid artery showing a mainly hypoechoic atherosclerotic plaque and the absence of the mobile thrombus (arrows). 
Due to the high embolization risk, the patient was hospitalised and prescribed Aspirin together with low molecular weight Heparin.

There was an improvement in the patient's neurological status. The control duplex scan evaluation, taken one week after the first examination revealed disappearance of the thrombus and persistence of the hypoechoic plaque (Figure 4). The patients was discharged on antiplatelet therapy.

\section{DISCUSSION}

A review of the literature showed that the incidence of a mobile thrombus, in studies using carotid ultrasound, ranges from 0.05 to $0.9 \%$ among stroke patients [3]. A mobile thrombus was most frequently found in the internal carotid artery (75\%), followed by the common carotid artery and carotid bifurcation (67\%) [2]. Bhatti et al., based on data from 145 cases (mean age 57.6 years; range 28 to 90 years) reported that this pathology was more prevalent in males. We have previously published a case of mobile thrombus in a 26-year-old male patient, localized in the right carotid bifurcation [1].

The aetiology of mobile thrombus can be cardioembolism, arterio-arterial embolism, dissection, complicated atherosclerotic plaque and hypercoagulable states $[2,3]$. The most frequently used diagnostic method is angiography (84\%) [2]. Nowadays, high resolution modern duplex ultrasound devices are widely accessible, improved with novel technologies including tissue harmonic imaging, with the added possibility of administrating a contrasting agent. They have a proven use in the treatment of the carotid pathology cited in this case report [4].

Due to the low incidence of mobile thrombus in the carotid arteries, there are no published or accepted guidelines regarding treatment. Some authors recommend conservative treatment (anti-aggregation, anticoagulation or both), while others consider this lesion to be a surgical emergency, needing immediate thrombectomy, due to the high embolization risk [1,2].

In a case series based on a literature review 30\% of patients were treated medically and $65 \%$ surgically [2]. In the medical group, complete dissolution of the thrombus occurred in $86 \%$ of cases. Conversely, complete resolution of the thrombus occurred in only $58 \%$ of the medically treated cases in another published case series [3].
Park JW et al. in their case series [4] combined successfully various endovascular approaches for the management of patients with free floating carotid thrombus. The placement of a self-expanding stent was effective in the stabilization of mobile thrombus, the utilization of distal antiembolic device was not mandatory. In case of mobile thrombus without any immobile portion they used suction thrombectomy. Arterial trapping, a more destructive intervention, was used in case of mobile thrombus, where the involved segment was too vast and was no more functional, but the embolization risk was high $[5,6]$.

\section{CONCLUSIONS}

The mobile carotid thrombus is detected usually incidentally on a carotid duplex ultrasound examination or angiography. Because the associated high embolisation risk, it represents a major neurological emergency. The presence of floating thrombus in a patient with clinical and imagistic evidence of stroke is a major therapeutical challenge for the neurologist. The presence of major stroke precludes active treatments as surgical intervention for thrombus removal of full anticoagulation therapy. The treatment strategies are not standardized and must be individualized, however in our case parenteral anticoagulation proved to be successful.

\section{- CONFLICT OF INTEREST}

The authors declare that they have no conflict of interest.

\section{DEFERENCES}

1. Bajko Z, Balasa R, Petrutiu S, Toma L, Russu S, Pascu I. Mobile carotid artery thrombus. Roman J Neurol. 2009;8(4):184-6.

2. Bhatti $A F$, Leon LR Jr, Labropoulos N, et al. Free-floating thrombus of the carotid artery: literature review and case reports. J Vasc Surg. 2007;45:199-205.

3. Chua HC, Lim T, Teo BC, Phua Z, Eng J. Free-floating thrombus of the carotid artery detected on carotid ultrasound in patients with cerebral infarcts: a 10-year study. Ann Acad Med Singapore. 2012;41:420-4.

4. Kemény $V$, Jung DK, Devuyst G. Ultrasound characteristics of adherent thrombi in the common carotid artery. Circulation. 2001;104:E24-5.

5. Schlachetzki $F$, Hoelscher $T$, Lange $M$, et al. Ultrasound finding of a mobile atheroma in the common carotid artery. Circulation. 2000;102:e105.

6. Park JW, Lee DH, Choi CG, Kim SJ, Suh DC. Various endovascular approaches to the management of Free Floating Carotid Thrombi. J Neurolntervent Surg. 2012;4(5):336-8. 